\title{
INFINITESIMAL CONDITIONS FOR THE EQUIVARIANCE OF MORPHISMS OF FIBERED MANIFOLDS
}

\author{
YVETTE KOSMANN-SCHWARZBACH
}

\begin{abstract}
ABSTRACr. We generalize the usual definition of the Lie derivative to the case of a morphism between fibered manifolds which does not necessarily preserve the base. We prove that the vanishing of the Lie derivatives is a necessary and sufficient condition for the equivariance of a morphism of fibered manifolds under the action of a connected Lie group.
\end{abstract}

0. Introduction. In many problems in mathematical physics one considers a 'field', in effect, a section of a fibered manifold (which is very often a vector bundle) over a base manifold representing space-time, and a group of transformations of the base. To know whether the field is physically meaningful, one asks the classical question, whether the group of transformations is a 'symmetry group' (in alternate terminology, an 'invariance group') of the field, that is, does there exist a lifting of the action of the group on the base manifold to an action on the fibered manifold by automorphisms (if the fibered manifold is a vector bundle one generally requires that the group act by vector-bundle automorphisms) such that the section under consideration be invariant under that action of the group? More generally, one can ask the same question regarding a differential operator from one fibered manifold to another over the same base manifold. One must consider liftings of the group action to the two fibered manifolds: if there exist liftings such that the differential operator is equivariant with respect to the two lifted actions, the group is called a 'symmetry group' (or an 'invariance group') of the differential operator or of the associated system of partial differential equations. (For scalar differential operators or, more generally, for differential operators on tensor bundles, the word 'invariant' is usually reserved for 'invariant under the natural liftings' while the word 'covariant' can be used in the other cases.) In general, one assumes that the group is a Lie group, considers its corresponding infinitesimal transformations, and determines whether the section or the differential operator is 'infinitesimally invariant'. In the case of a section that means, does there exist a lifting of the Lie algebra of vector fields on the base to a Lie algebra of infinitesimal automorphisms of the fibered manifold such that the section is infinitesimally invariant, i.e., such that its Lie derivatives with respect to those vector fields defined by this lifting vanish?

Received by the editors March 6, 1978 and, in revised form, June 13, 1978.

AMS (MOS) subject classifications (1970). Primary 53A55; Secondary 50A15, 22A60.

Key words and phrases. Lie group of transformations, Lie derivative, morphism of fibered manifolds, equivariant morphism, geometric objects, natural bundles. 
For a differential operator one asks whether there exist liftings of the Lie algebra of vector fields such that the differential operator is infinitesimally equivariant. This is the infinitesimal version of the classical similarity methods for partial differential equations. One way of solving the question is to define the Lie derivatives of the differential operator associated with the liftings of the vector fields in the Lie algebra, and to write the conditions for the vanishing of those Lie derivatives. In the present paper we give a definition of the Lie derivative of a morphism between fibered manifolds which does not necessarily preserve the base, and we thus obtain generalizations of the similarity methods.

1. Group actions on fibered manifolds and Lie derivatives of sections. Let $\pi$ : $E \rightarrow M$ denote a smooth locally trivial fibered manifold. Let $G$ be a Lie group. We assume that $G$ acts on $M$ by diffeomorphisms and that the action $\mu \rightarrow \mu_{M}$ of $G$ on $M$ lifts to an action $\mu \rightarrow \mu_{E}$ of $G$ on $E$ by fiber-preserving diffeomorphisms. Moreover, we require the condition (C): given a oneparameter family $t \rightarrow \mu_{t}$ in $G$ such that $(t, x) \in \mathbf{R} \times M \rightarrow\left(\mu_{t}\right)_{M} x \in M$ is smooth, then $(t, y) \in \mathbf{R} \times E \rightarrow\left(\mu_{t}\right)_{E} y \in E$ is smooth. Examples of this situation are: all trivial fibrations, all bundles of tensors and bundles of jets of tensors over $M$, frame bundles and higher-order frame bundles over $M$ and bundles of forms over these (bundles of connections), with $G$ a Lie group of transformations of $M$; also all bundles of tensor-spinors over a spin manifold $M$ when $G$ is a closed subgroup of the connected component of the conformal group of $M$.

We will use the terms functions and sections to mean smooth functions and smooth sections. $G$ acts on the ring of functions $f$ over $M$ by

$$
\mu_{M} \cdot f=f \circ \mu_{M}^{-1} \quad(\text { for } \mu \text { in } G) \text {. }
$$

$G$ acts on the set of sections $\psi$ of $E$ by

$$
\mu_{E} \cdot \psi=\mu_{E} \circ \psi \circ \mu_{M}^{-1} \quad(\text { for } \mu \text { in } G) \text {. }
$$

More generally, $G$ acts on the set of local sections of $E$.

Let $X$ be in the Lie algebra $g$ of $G$. We set $\mu_{t}=\exp (t X)$, for $t$ real. We first recall the definition of Kuiper and Yano [1, p. 419] which is also that of Salvioli $[4,4.3]$ up to sign:

(1.3) Definition. The Lie derivative of a local section $\psi$ of $E$ with respect to $X$ is the local field $X_{E} \cdot \psi$ of vertical tangent vectors to $E$ along $\psi$ defined by

$$
\left(X_{E} \cdot \psi\right)_{x}(h)=\left.\frac{d}{d t} h\left(\left(\left(\mu_{t}\right)_{E} \cdot \psi\right)(x)\right)\right|_{t=0}
$$

where $h$ is a function on $E$ and $x$ is a point in the domain of $\psi$.

It is clear that $X_{E}$ is a first-order differential operator from $E$ into the vertical tangent bundle of $E$.

Assume that $G$ is a connected Lie group. A local section $\psi$ of $E$ is invariant under $G$ if and only if $X \cdot \psi=0$ for all $X$ in the Lie algebra $g$ of $G$. It is this property that we wish to extend to a more general situation. 
2. Lie derivatives of morphisms. Let $\pi: F \rightarrow M$ and $\pi^{\prime}: F^{\prime} \rightarrow M^{\prime}$ be two fibered manifolds as above. We denote by $\mu \rightarrow \mu_{M}, \mu \rightarrow \mu_{F}, \mu \rightarrow \mu_{M^{\prime}}^{\prime}$ and $\mu \rightarrow \mu_{F}^{\prime}$, the actions of $G$ on $M, F, M^{\prime}$ and $F^{\prime}$. $G$ acts on the set of local morphisms $u$ from $F$ to $F^{\prime}$ by

$$
\mu \cdot u=\mu_{F^{\prime}}^{\prime} \circ u \circ \mu_{F}^{-1} \quad(\text { for } \mu \text { in } G) .
$$

Note that if $u$ projects onto the mapping $u_{M}$ from $M$ into $M^{\prime}$, then $\mu \cdot u$ projects onto $\mu_{M^{\prime}}^{\prime} \circ u_{M} \circ \mu_{M}^{-1}$. In particular, if $u$ projects onto a local diffeomorphism of $M$ into $M^{\prime}$, so does $\mu \cdot u$ for any $\mu$ in $G$.

Let $v$ be a local morphism from $F$ to $F^{\prime}$ which projects onto a local diffeomorphism $v_{M}$ from $M$ to $M^{\prime}$ and let $\psi$ be a local section of $F$; then $v \cdot \psi$ denotes the local section of $F^{\prime}$ defined by

$$
v \cdot \psi=v \circ \psi \circ v_{M}^{-1} .
$$

(2.3) Definition. Let $u$ be a local morphism from $F$ to $F^{\prime}$ which projects onto a local diffeomorphism $u_{M}$ from $M$ into $M^{\prime}$. Let $X$ be in the Lie algebra $\mathfrak{g}$ of $G$. The Lie derivative $X \cdot u$ of $u$ with respect to $X$ is defined by

$$
((X \cdot u) \psi)_{x^{\prime}}(h)=\left.\frac{d}{d t} h\left(\left(\left(\mu_{t} \cdot u\right) \cdot \psi\right)\left(x^{\prime}\right)\right)\right|_{t=0}
$$

where $\psi$ is a local section of $F, h$ is a function on $F^{\prime}$, and $x^{\prime}$ is a point of the domain of $u \cdot \psi$.

Let us denote by $V F^{\prime}$ the vertical tangent bundle to $F^{\prime}$, considered as a bundle over $M^{\prime}$. $((X \cdot u) \psi)_{x^{\prime}}$ is a vertical tangent vector to $F^{\prime}$ at the point $(u \cdot \psi)\left(x^{\prime}\right) \cdot(X \cdot u) \psi$ is a section of $V F^{\prime}$ over $M^{\prime}$ and it can be considered as a section of the induced bundle $u_{M}^{*}\left(V F^{\prime}\right)$ over $M$. As such the value of $(X \cdot u) \psi$ at $x$ in $M$ depends only upon the value of $\psi$ and its first derivatives at $x$. Whence

(2.5) Proposition. The Lie derivative $X \cdot u$ of $u$ with respect to $X$ is a first-order differential operator from $F$ to $u_{M}^{*}\left(V F^{\prime}\right)$.

Let us now examine the case of a base-preserving morphism.

(2.6) Proposition. Assume that $M=M^{\prime}$. When u projects onto the identity of an open set of $M$, and the actions of $G$ on $F$ and $F^{\prime}$ project onto the same action on $M$, then $X \cdot u$ is a lifting of $u$ into $V F^{\prime}$.

In fact, under these hypotheses, $((X \cdot u) \psi)_{x}$ depends only on the value $y$ of $\psi$ at $x$ and not on its first derivatives. For $y$ in $F,(X \cdot u)_{y}$ is the vector in $V_{u(y)} F^{\prime}$ defined by

$$
(X \cdot u)_{y}(h)=\left.\frac{d}{d t} h\left(\left(\mu_{t} \cdot u\right)(y)\right)\right|_{t=0}
$$

where $h$ is a function on $F^{\prime}$.

We remark that if $M=M^{\prime}$ and $F=M$, then a local section of $F^{\prime}$ can be viewed as a local morphism from $F$ into $F^{\prime}$ over the identity of an open set of $M$. (1.2) is then a particular case of (2.1) and it is clear from (2.7) that 
Definition (2.3) is indeed a generalization of (1.3).

(2.8) Lemma. For $\mu$ in $G$, denote by $T \mu_{F^{\prime}}^{\prime}$ the differential of $\mu_{F^{\prime}}^{\prime}$. Then

$$
\left(T \mu_{F^{\prime}}^{\prime}\right)((X \cdot u) \psi)=\left(\left(T \mu_{M} X\right) \cdot(\mu \cdot u)\right) \psi
$$

The proof is a straightforward computation.

(2.9) LEMMA. For s real,

$$
\left(T\left(\mu_{s}\right)_{F^{\prime}}^{\prime}((X \cdot u) \psi)\right)_{x^{\prime}}(h)=\left(X \cdot\left(\mu_{s} \cdot u\right) \psi\right)_{x^{\prime}}(h)=\frac{d}{d s} h\left(\left(\left(\mu_{s} \cdot u\right) \cdot \psi\right)\left(x^{\prime}\right)\right) \text {. }
$$

The proof follows from the equaliy $\mu_{t+s} \cdot u=\mu_{t} \cdot\left(\mu_{s} \cdot u\right)$ and from Lemma (2.8).

We now state the infinitesimal conditions for the equivariance of a morphism of fibered manifolds $F$ and $F^{\prime}$ under a Lie group of transformations acting on $F$ and $F^{\prime}$.

(2.10) TheOREM. Assume that $G$ is a connected Lie group. A local morphism $u$ from $F$ to $F^{\prime}$ which projects onto a local diffeomorphism from $M$ to $M^{\prime}$ is equivariant with respect to $G$ if and only if $X \cdot u=0$ for all $X$ in the Lie algebra $g$ of $G$.

Proof. The necessity of the condition is clear. Assume $X \cdot \boldsymbol{u}=\mathbf{0}$ for some $X$ in g. By Lemma (2.9), $d h\left(\left(\left(\mu_{s} \cdot u\right) \cdot \psi\right)\left(x^{\prime}\right)\right) / d s=0$ identically. Thus for any local section $\psi$, the local section $\left(\mu_{s} \cdot u\right) \cdot \psi$ must be independent of $s$, and therefore equal to $u \cdot \psi$. This in turn implies that $\mu_{s} \cdot u=u$ for all $s$, which means that $u$ is equivariant with respect to $\mu_{s}$. If $X \cdot u=0$ for all $X$ in $g, u$ is equivariant under the connected component of $G$, whence the theorem.

We denote by $V u$ the restriction to the vertical tangent bundle $V F$ of $F$ of the differential $T u$ of $u$. It is a vector-bundle morphism from $V F$ to $V F^{\prime}$. By (2.2) it maps local sections of $V F$ over $M$ into local sections of $V F^{\prime}$ over $M^{\prime}$. It is clear that for a local section $(\psi, \xi)$ of $V F$,

$$
(V u \cdot(\psi, \xi))_{x^{\prime}}=\left.\frac{d}{d t}\left(u \cdot \psi_{t}\right)\left(x^{\prime}\right)\right|_{t=0}
$$

where $\psi_{t}$ is a local one-parameter family of local sections of $F$ such that $\psi_{0}=\psi$ and $d \psi_{t}(x) /\left.d t\right|_{t=0}=\xi_{x}$ for $x$ in the domain of $\xi$, and $x^{\prime}$ is a point in $M^{\prime}$ such that both sides are defined.

(2.12) Proposition. The Lie derivative $X \cdot u$ of $u$ with respect to $X$ satisfies

$$
X \cdot u=X_{F}^{\prime} \circ u-V u \circ X_{F}
$$

where $X_{F}$ (resp. $X_{F}^{\prime}$ ) is the first-order differential operator from $F$ into $V F$ (resp. from $F^{\prime}$ into $\left.V F^{\prime}\right)$ defined by (1.4), and $u$ (resp. $V u$ ) acts on the local sections of $F($ resp. $V F)$ by (2.2). 
Proof. We verify that both sides of (2.13) act in the same way on a local section $\psi$ of $F$, using the fact that $u$ is continuous on each fiber of $F$, and relation (2.11) for $(\psi, \xi)=X_{F} \cdot \psi$.

The right-hand side of (2.13) is meaningful in the more general case of a local morphism $u$ from the $k$ th jet bundle (for some $k>0$ ) of $F$ into $V F^{\prime}$. We call $u$ a generalized differential operator of order $k$ from $F$ into $F^{\prime} . V u$ is then a mapping from the local sections of $V F$ over $M$ into the local sections of $V F^{\prime}$ over $M^{\prime}$ defined by formula (2.11) where $u \cdot \psi_{t}$ denotes the action of $u$ on the $k$ th jet of $\psi_{t}$ at a point. Thus (2.13) provides a definition of the Lie derivative $X \cdot u$ in this more general case. We need this extension in order to state Proposition (2.14) concerning the Lie derivation with respect to the bracket of two vector fields, since $X \cdot u$ is a generalized differential operator of order 1 , while $u$ itself is of order 0 . (A morphism from $F$ to $F^{\prime}$ can be regarded as a morphism from $F$ to $V F^{\prime}$.)

(2.14) Proposition. The Lie derivation with respect to the bracket of two vector fields is the commutator of the Lie derivations. More precisely, for any two elements $X$ and $Y$ in the Lie algebra $\mathfrak{g}$, and for any local morphism $u$ from $F$ to $F^{\prime}$ which projects onto a local diffeomorphism of $M$ into $M^{\prime}$,

$$
[X, Y] \cdot u=X \cdot(Y \cdot u)-Y \cdot(X \cdot u) \text {. }
$$

Proof. $X$ and $Y$ act on the sections of $F$; they also act on the sections of $V F$ and $[X, Y]_{F} \cdot \psi=X_{V F} \cdot\left(Y_{F} \cdot \psi\right)-Y_{V F} \cdot\left(X_{F} \cdot \psi\right)$. Thus $X \rightarrow X_{F}$ is a Lie algebra homomorphism from $g$ into a Lie algebra of differential operators from $F$ to $V F$. (That Lie algebra will be studied in detail elsewhere.) In the same way $X \rightarrow X_{F}^{\prime}$ is a Lie algebra homomorphism. Formula (2.15) follows from those two facts and from (2.13).

If $F^{\prime}$ is a vector bundle, the set of all generalized differential operators from $F$ into $F^{\prime}$ is a vector space, filtered by the order.

(2.16) COROllary. Assume that $F^{\prime}$ is a vector bundle. The Lie derivation is a Lie algebra homomorphism from $g$ into a Lie algebra of endomorphisms of degree 1 of the filtered vector space of all generalized differential operators from $F$ into $F^{\prime}$. In other words, the vector space of generalized differential operators from $F$ to $F^{\prime}$ is a representation space for $\mathrm{g}$.

This follows from the above definitions and from Proposition (2.14) extended to generalized differential operators.

In the next paragraph we point out the simplifications that occur in the case of vector bundles.

3. The case of vector bundles. If $E$ is a vector bundle over $M$, the vertical tangent bundle of $E$ is identified with $E \times E$, and by Definition (1.3), the Lie derivative with respect to $X$ of a local section $\psi$ is the local section

$$
\left(X_{E} \cdot \psi\right)_{x}=\left.\frac{d}{d t}\left(\left(\left(\mu_{t}\right)_{E} \cdot \psi\right)(x)\right)\right|_{t=0}
$$


$X_{E}$ is a first-order differential operator on $E$ with values in $E$, and it is linear when $G$ acts on $E$ by vector-bundle automorphisms. More generally, assume in Definition (2.3) that $F^{\prime}$ is a vector bundle and that $V F^{\prime}$ is identified with $F^{\prime} \times F^{\prime}$. Then $X \cdot u$ satisfies

$$
((X \cdot u) \psi)_{x^{\prime}}=\left.\frac{d}{d t}\left(\left(\left(\mu_{t} \cdot u\right) \cdot \psi\right)\left(x^{\prime}\right)\right)\right|_{t=0}
$$

where the derivative is taken in the fiber of $F^{\prime}$ at $x^{\prime} . X \cdot u$ is a first-order differential operator from $F$ to $u_{M}^{*} F^{\prime}$. Proposition (2.12) takes the following form:

(3.3) Proposition. Let $F$ and $F^{\prime}$ be vector bundles and let $u$ be a local vector-bundle morphism from $F$ to $F^{\prime}$ which projects onto a local diffeomorphism of $M$ into $M^{\prime}$. The Lie derivative of $u$ with respect to $X$ is

$$
X \cdot u=X_{F^{\prime}}^{\prime} \circ u-u \circ X_{F}
$$

where $X_{F}$ and $X_{F}^{\prime}$ act as first-order differential operators on the local sections of $F$ and $F^{\prime}$ respectively by (3.1), and $u$ acts linearly on the sections of $F$ by (2.2).

Proof. When $V F$ is identified with $F \times F$, and when $u$ is linear on each fiber, $V u$ can be identified with $u$. Thus (3.4) follows from (2.13).

(3.5) ExAMPLE. Let us assume $M=M^{\prime}, F=T M=F^{\prime}$, and let us compute $X \cdot T v$ where $v$ is a diffeomorphism of $M$ and $X$ is a vector field on $M$. For a local section $Y$ of $T M, X_{T M} \cdot Y=-[X, Y]$ and therefore

$$
\begin{aligned}
(X \cdot T v)(Y) & =\left(X_{T M} \circ T v\right)(Y)-\left(T v \circ X_{T M}\right)(Y) \\
& =-[X, T v(Y)]+T v([X, Y]) \\
& =-[X, T v(Y)]+[T v(X), T v(Y)] \\
& =[T v(X)-X, T v(Y)],
\end{aligned}
$$

whence

$$
X \cdot T v=\operatorname{Ad}(T v(X)-X) \circ T v .
$$

In particular, it is clear that, because $T v$ is assumed to be invertible, the vector field $X$ is invariant under $v$ if and only if $X \cdot T v=0$.

(3.6) Example. Assume in Proposition (3.3) that $M=M^{\prime}, u$ projects onto the identity of an open set of $M$ and the actions of $G$ on $F$ and $F^{\prime}$ project onto the same action on $M$. By Proposition (2.6) $X \cdot u$ is a local morphism from $F$ to $F^{\prime}$. If $G$ acts on $F$ and $F^{\prime}$ by vector-bundle automorphisms, $X \cdot u$ is a local vector-bundle morphism and it is the Lie derivative with respect to $X$ of $u$ considered as a local section of $F^{*} \otimes F^{\prime}$, where $F^{*}$ is the dual of $F$.

(3.7) Example. Assume in Proposition (3.3) that $M=M^{\prime}, F$ is the vectorbundle of $k$-jets of sections of a vector bundle $E$ over $M$. A vector-bundle morphism $u$ from $F$ to $F^{\prime}$ which projects onto the identity of $M$ is a linear differential operator of order $k$ from $E$ to $F^{\prime}$. Its Lie derivative with respect to $X$ is a differential operator of order $\leqslant k+1$. It is linear if $G$ acts on $F$ and $F^{\prime}$ by vector-bundle automorphisms. 
4. Lie derivatives in bundles of geometric objects. Since the definition of the Lie derivatives only involves the local behavior of the transformations defined by the group $G$, we can extend the definition to the case of pseudo-groups. Let us consider a transitive pseudo-group $\Lambda$ of transformations of a manifold $M$. The existence of liftings of those local diffeomorphisms of $M$ which belong to $\Lambda$ to local automorphisms of a fibered manifold $E$ over $M$ is the essential requirement for $E$ to be a 'geometric object bundle' over $M$ [1, Theorem 3] or a 'natural bundle' over $M([2$, p. 464], [3, p. 323]) or a 'bundle of geometric objects' over $M$ [4, Definition 1.3]. (In [2], [3] and [4], the pseudo-group under consideration is the pseudo-group of all local diffeomorphisms of $M$.) We shall say that a fibered manifold $\pi: E \rightarrow M$ is a 'bundle of $\Lambda$-geometric objects' over $M$ if every $\mu$ in $\Lambda$ lifts to a local automorphism $\mu_{E}$ of $E$ over $\mu$, if this lifting is compatible with the restriction mappings and with the law of composition and if condition (C) of $\$ 1$ is satisfied.

If $F$ and $F^{\prime}$ are two bundles of $\Lambda$-geometric objects over the same manifold $M$, if $X$ is a local vector field on $M$, and $X$ generates a flow in the pseudo-group $\Lambda$, we can still define by formula (2.4) the Lie derivative of a local morphism from $F$ to $F^{\prime}$ with respect to $X$. Theorem $(2.10)$ can then be modified as follows: If $\left(\mu_{t}\right)$ is the flow of $X$, then $u$ is equivariant with respect to $\mu_{t}$ (for those $t$ for which it is defined) if and only if $X \cdot u=0$. Also, a necessary condition for $u$ to be equivariant with respect to $\Lambda$ is that $X \cdot u=0$ for all vector fields $X$ which generate a flow in $\Lambda$. In particular, using the terminology of $[3, \S 3]$,

(4.1) Proposition. A necessary condition for a morphism $u$ from a natural bundle $F$ into a natural bundle $F^{\prime}$ to be natural is that $X \cdot u=0$ for all local vector fields $X$ on $M$.

\section{REFERENCES}

1. N. Kuiper and K. Yano, On geometric objects and Lie groups of transformations, Nederl. Akad. Wetensch. Proc. Ser. A 58 (1955), 411-420.

2. A. Nijenhuis, Geometric aspects of formal differential operators on tensor fields, Proc. Internat. Congr. Math. (Edinburgh, 1958), Cambridge Univ. Press, Cambridge, 1960, pp. 463-469.

3. __ Natural bundles and their general properties, Differential Geometry, in honor of $\mathbf{K}$. Yano, edited by S. Kobayashi, M. Obata and T. Takahashi, Kinokuniya, Tokyo, 1972, pp. 317-334.

4. S. Salvioli, On the theory of geometric objects, J. Differential Geometry 7 (1972), 257-278.

Department of Mathematics, New York Institute of Technology, Old Westbury, LONG IsLAND, NeW YORK 11568

Current address: Department of Mathematics, Brooklyn College (CUNY), Brooklyn, New York 11210 